This article is licensed under the Creative Commons Attribution-NonCommercial 4.0 International License (CC BY-NC) (http://www.karger.com/Services/OpenAccessLicense). Usage and distribution for commercial purposes requires written permission.

\title{
Clinical Efficacy and Safety of the EX-PRESS Filtration Device in Patients with Advanced Neovascular Glaucoma and Proliferative Diabetic Retinopathy
}

\author{
Rana Hanna $^{a} \quad$ Beatrice Tiosano $^{\mathrm{a}} \quad$ Shmuel Graffi ${ }^{\mathrm{b}} \quad$ Dan Gaton $^{\mathrm{c}}$ \\ a Department of Ophthalmology, Hillel Yaffe Medical Center, affiliated to the Rappaport \\ Faculty of Medicine, The Technion, Hadera, Israel; ${ }^{b}$ Department of Ophthalmology, Baruch \\ Padeh Medical Center, affiliated to the Faculty of Medicine, Bar Ilan University, \\ Galilee, Israel; ${ }^{C}$ Department of Ophthalmology, Rabin Medical Center, Beilinson Hospital, \\ affiliated to the Sackler Faculty of Medicine, Tel Aviv University, Tel Aviv, Israel
}

\section{Keywords}

EX-PRESS device $\cdot$ Neovascular glaucoma $\cdot$ Proliferative diabetic retinopathy

\begin{abstract}
Background: The prognosis of conventional filtration surgery in eyes with neovascular glaucoma (NVG) is limited due to increased fibrovascular proliferation or bleeding. This study aims to evaluate the safety and efficacy of the EX-PRESS filtration device in the management of NVG associated with proliferative diabetic retinopathy (PDR). Methods: In this retrospective case series, we reviewed the medical records of patients diagnosed as having NVG associated with PDR who underwent EX-PRESS filtration surgery. The main outcome measures were: postoperative intraocular pressure (IOP), the percent of IOP drop, the number of glaucoma medications, visual acuity, and complications of surgery. Successful surgery was de-
\end{abstract}




\section{Case Reports in Ophthalmology}

Case Rep Ophthalmol 2018:9:61-69

DOI: $10.1159 / 000479363$

(C) 2018 The Author(s). Published by S. Karger AG, Basel www.karger.com/cop

Hanna et al.: Clinical Efficacy and Safety of the EX-PRESS Filtration Device in Patients with Advanced Neovascular Glaucoma and Proliferative Diabetic Retinopathy

fined as an IOP $<22 \mathrm{~mm} \mathrm{Hg}$ and $>5 \mathrm{~mm} \mathrm{Hg}$ with or without additional glaucoma surgery, and no loss of light perception or less than a 2-line decrease on the Snellen chart of the best corrected visual acuity (BCVA). Results: Five patients (5 eyes) were included in this study. The mean preoperative IOP was $33.4 \pm 5.9 \mathrm{~mm} \mathrm{Hg}$ compared to an IOP of $17.0 \pm 3.0 \mathrm{~mm} \mathrm{Hg}$ at the last follow-up $(p=0.003)$. The mean number of preoperative anti-glaucoma medications was $3.8 \pm 0.4$ compared to $2.2 \pm 1.5(p=0.06)$ at the last follow-up visit. Final visual acuity improved or stabilized within 1 Snellen line in all 5 patients. Three patients had a "hypertensive phase" (defined as an IOP $>21 \mathrm{~mm} \mathrm{Hg}$ during the first 6 postoperative months) which resolved within 2 months. Two patients developed a hyphema that resolved spontaneously. None of the patients experienced any serious complications. Conclusion: EX-PRESS filtration device has a good IOP-lowering effect and a low rate of complications in patients with advanced NVG associated with PDR. In addition, there was no loss of light perception or no line decrease of the BCVA.

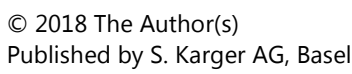

\section{Introduction}

Neovascular glaucoma (NVG) is a refractory disease. It has been reported to occur in over 40 ocular diseases, most commonly, diabetes mellitus, central retinal vein occlusion, and ocular ischemic syndrome [1,2]. Fibrovascular proliferation in the anterior segment may obstruct the trabecular meshwork and cause peripheral anterior synechiae, progressively closing the anterior chamber angle and leading to an intractable elevation of the intraocular pressure (IOP). Ischemia triggers the release of vascular endothelial growth factor (VEGF), interleukin, and other angiogenic factors that can diffuse into the anterior segment, causing neovascularization of the iris and anterior chamber angle [3-5]. IOP control in NVG patients often necessitates surgical treatment, since medical management alone, such as IOP-lowering medications and anti-VEGF injections, together with panretinal photocoagulation (PRP) are often inadequate. However, conventional filtration surgery carries a guarded prognosis in eyes with NVG due to increased fibrovascular proliferation or bleeding. Allen et al. [6] reported that trabeculectomy surgeries had a $67 \%$ success rate after a mean follow-up of 22 months. Glaucoma drainage implants, such as the Ahmed glaucoma valve (AGV; New World Medical, Rancho Cucamonga, CA, USA), especially in combination with mitomycin C (MMC), have been used for the treatment of NVG in patients with proliferative diabetic retinopathy (PDR), forming an alternate aqueous pathway that promotes bleb formation far from the limbus. Although the AGV implant is successful in providing early and intermediate IOP control, it fails to achieve control of IOP in patients with NVG in the long term (5 years) [7-12].

The EX-PRESS filtration device is a small non-valved device consisting of a stainless steel, magnetic resonance imaging-compatible implant. It has a $50-\mu \mathrm{m}$ and a $200-\mu \mathrm{m}$ lumen that lowers IOP by shunting aqueous humor from the anterior chamber into the subconjunctival area near the limbus $[13,14]$. To the best of our knowledge, there are no reports on the safety and efficacy of the EX-PRESS filtration device in the management of NVG in eyes with a history of PDR. The purpose of this study is to report our experience and the out- 


\section{Case Reports in Ophthalmology}

Case Rep Ophthalmol 2018;9:61-69

DOI: $10.1159 / 000479363$

(C) 2018 The Author(s). Published by S. Karger AG, Basel www.karger.com/cop

Hanna et al.: Clinical Efficacy and Safety of the EX-PRESS Filtration Device in Patients with Advanced Neovascular Glaucoma and Proliferative Diabetic Retinopathy

comes of EX-PRESS filtration device surgery with the addition of MMC application in patients with NVG.

\section{Methods}

This retrospective case series included 5 eyes of 5 consecutive advanced glaucoma patients diagnosed as having NVG due to PDR who underwent EX-PRESS filtration device surgery between September 2011 and May 2014. An indication for surgery was failed maximal glaucoma medication treatment in advanced NVG patients (defined by a progression of retinal nerve fiber layer thinning on 3 successive visits). The diagnosis of NVG was made by a glaucoma specialist (B.T.) and defined as neovascularization of the iris and/or iridocorneal angle (the latter diagnosed by gonioscopy), with an IOP $>21 \mathrm{~mm} \mathrm{Hg}$. Advanced glaucoma was defined according to ICD-9 staging codes, which include elevated IOP, optic nerve abnormalities consistent with glaucoma, glaucomatous visual field abnormalities in both hemifields, and/or loss within 5 degrees of fixation in at least 1 hemifield [15]. Exclusion criteria were patients younger than 18 years, and those with a history of cyclodestructive procedures, the presence of other retinal diseases, and a follow-up of $<12$ months.

The study was approved by the Hillel Yaffe Medical Center's Institutional Review Board, and it was carried out in accordance with The World Medical Association's Declaration of Helsinki. All EX-PRESS surgeries were performed by a single surgeon (B.T.). Two patients underwent EX-PRESS $50-\mu \mathrm{m}$ implantation and 3 patients underwent EX-PRESS $200-\mu \mathrm{m}$ implantation. One patient also underwent cataract extraction at the time of surgery. One patient had undergone trabeculectomy 2 years before the EX-PRESS surgery.

All 5 patients underwent PRP prior to surgery. Two patients underwent vitrectomy surgery because of vitreous hemorrhage and a retained nucleus in the vitreous after a complicated cataract surgery 3 and 6 months before the index EX-PRESS surgery, respectively. All of the patients received an intravitreal injection of bevacizumab at least 3 months before the EX-PRESS surgery.

The data that were collected during each visit were age, gender, and the results of a complete ophthalmological evaluation, including visual acuity, preoperative IOP, the number of glaucoma medications, previous bevacizumab injections, and previous intraocular surgeries.

The follow-up schedule consisted of visits on the first postoperative day, and at 1 week, 1 month, and every 3-6 months after surgery until a maximal follow-up of 15 months after surgery. A postoperative "hypertensive phase" was defined as an IOP $>21 \mathrm{~mm}$ Hg during the first 6 postoperative months.

Surgical success was defined as an IOP $<22$ and $>5 \mathrm{~mm} \mathrm{Hg}$ without additional glaucoma surgery and with no loss of light perception (LP) on at least 2 postoperative visits. Surgical failure was defined as an IOP $<5$ or $>22 \mathrm{~mm} \mathrm{Hg}$ on at least 2 consecutive follow-up visits, a deterioration of the best corrected visual acuity (BCVA) with loss of LP, or the need for additional glaucoma surgical interventions. Subjects who had a decrease in their BCVA but maintained LP vision or greater were not considered a surgical failure. The definition of hypotony in this study was an IOP of $\leq 5 \mathrm{~mm} \mathrm{Hg}$ at 2 consecutive visits. 


\section{Case Reports in Ophthalmology}

Case Rep Ophthalmol 2018;9:61-69

DOI: $10.1159 / 000479363$

(C) 2018 The Author(s). Published by S. Karger AG, Basel www.karger.com/cop

Hanna et al.: Clinical Efficacy and Safety of the EX-PRESS Filtration Device in Patients with Advanced Neovascular Glaucoma and Proliferative Diabetic Retinopathy

The surgical technique consisted of a fornix-based conjunctival incision with subconjunctival anesthesia. A wound-modulating agent (MMC 0.02\%; Kyowa Hakko Kirin Co. Ltd., Japan) was applied under the conjunctiva for $1 \mathrm{~min}$ and copiously irrigated with balanced salt solution. A half-thickness trapezoidal scleral flap of $4 \times 4 \mathrm{~mm}$ was constructed and advanced anteriorly into the cornea. A 25-gauge needle was used to penetrate the anterior chamber, and the EX-PRESS glaucoma device was inserted into the anterior chamber through this entrance. The corneoscleral flap was sutured at its 2 corners using a 10-0 nylon suture. The conjunctival flap was advanced and sutured by a continuous 10-0 nylon suture. Cefuroxime sodium (Zinacef $750 \mathrm{mg} / 50 \mathrm{~mL}$; GlaxoSmithKline, Italy) was injected into the anterior chamber at the end of the procedure. Following surgery, all patients received the topical antibiotic ofloxacin $0.3 \%$ (Oflox; Allergan, Ireland) 5 times daily for 1 week. Steroid eye drops were prescribed 5 times daily for 4 weeks, followed by tapering down over 1 month. They consisted of a combined ophthalmic suspension containing dexamethasone $0.1 \%$, neomycin sulfate 3,500 IU/mL, and polymyxin B sulfate 6,000 IU/mL (Maxitrol; AlconCouvreur, Belgium).

\section{Statistics}

The values are presented as means \pm standard deviation (SD) and percentages. The differences between the groups were compared using the one-way ANOVA test. A $p$ value of $<0.05$ was considered to be statistically significant. The statistical analysis was carried out using the SPSS-23 statistical software (IBM Corp, Armonk, NY, USA).

\section{Results}

The demographic data and preoperative information of the 5 study patients are listed in Table 1. The postsurgical follow-up period was 12-15 months. One patient who had a follow-up visit 2 months after surgery, and another follow-up visit 15 months after surgery was included. All patients were under individualized maximal therapy before surgery. The mean preoperative IOP was $33.4 \pm 5.9 \mathrm{~mm} \mathrm{Hg}$ (range $26-40 \mathrm{~mm} \mathrm{Hg}$ ), and it decreased to $17.0 \pm 3.0$ $\mathrm{mm} \mathrm{Hg}(12-20 \mathrm{~mm} \mathrm{Hg})(p=0.003)$. There was a mean of $3.8 \pm 0.4$ glaucoma medications before surgery which dropped to $2.2 \pm 1.1$ postoperatively $(p=0.06)$ (Table 2). Final visual acuity improved or stabilized within 1 Snellen line in all 5 patients.

The IOP was lowered in all of the patients on the first postoperative day, with the mean IOP measuring $10.8 \pm 5.2 \mathrm{~mm} \mathrm{Hg}$ without additional glaucoma medications ( $p=0.001$ ). None of the patients developed postoperative hypotony. Three patients had a "hypertensive phase" that gradually stabilized over 2 months: their IOP was controlled with glaucoma medications during that period. Surgical success was achieved in all of the patients at the last follow-up (Fig. 1). Figure 2 shows the IOP measurements for each patient until final follow-up.

Two patients developed intraoperative hyphema that resolved spontaneously within the first week of follow-up. There were no major intraoperative, early, or late postoperative complications. 


\section{Case Reports in Ophthalmology}

Case Rep Ophthalmol 2018;9:61-69

DOI: $10.1159 / 00047936$

(C) 2018 The Author(s). Published by S. Karger AG, Basel www.karger.com/cop

Hanna et al.: Clinical Efficacy and Safety of the EX-PRESS Filtration Device in Patients with Advanced Neovascular Glaucoma and Proliferative Diabetic Retinopathy

\section{Discussion}

The purpose of this study was to analyze the clinical outcome of EX-PRESS filtration surgery in patients with NVG associated with PDR. There are only a few reports in the literature on glaucoma drainage devices for the treatment of NVG [7-12]. Glaucoma drainage implants, especially the AGV, in combination with an injection of bevacizumab are gaining wide acceptance as a primary procedure for patients with NVG. Variable success rates for IOP control have been reported: Mahdy et al. [16] reported a 95\% success rate (complete and qualified) using AGV surgery for NVG when intravitreal bevacizumab injection and PRP were followed by implantation of an AGV. Ma et al. [17] reported a 70\% success rate 1 year after AGV implantation combined with an intraoperative bevacizumab injection. A recent study reported a success rate of $79 \%$ after 1 year and of $56 \%$ after 2 years using the Baerveldt implant for treating NVG [18]. The success rates when using the Molteno tube were reported to be $37-72 \%$ and $29-60 \%$ after 1 and 2 years, respectively $[8,19,20]$.

The EX-PRESS glaucoma implant is a well-tolerated miniature glaucoma device aimed at lowering IOP. Previous publications have reported on the device's efficacy and relatively low rates of postoperative complications [21,22]. Our results show that the control of IOP was achieved up to the final follow-up visit in all of our patients with NVG associated with PDR, although 4 of them still required glaucoma medications. The overall mean number of glaucoma medications for the group, however, was reduced (Table 2). There was only 1 intraoperative minor and transient complication (mild hyphema) in 2 patients, and no postoperative complications in any patient.

A period of transient elevation of IOP ("hypertensive phase") was described after glaucoma drainage implant surgery by Ayyala et al. [9]. Those authors reported that $83.5 \%$ of their patients with advanced uncontrolled glaucoma that underwent AGV implantation experienced a "hypertensive phase" compared to $43.5 \%$ of the patients that underwent double-plate Molteno implantation [23]. Three of our 5 patients also developed a transient "hypertensive phase".

Many reports have shown the efficacy of anti-VEGF application in treating NVG [24, 25]. Intravitreal and intracameral bevacizumab injections have been reported to be a safe and effective adjuvant for glaucoma drainage devices in the setting of NVG $[16,17]$. In the current study, all 5 patients received an intravitreal bevacizumab injection within 1 month before surgery.

The limitations of this study include its small sample size as well as its retrospective nature, and the lack of a control group. In addition, although all of the patients showed surgical success, we could not properly evaluate the effect of the bevacizumab injection alone because of the small sample size. The study size also prevented a comparison of the surgical results between the 50- $\mu \mathrm{m}$ lumen EX-PRESS device and the 200- $\mu \mathrm{m}$ lumen EX-PRESS device, and there are no studies comparing the efficacy of $50-\mu \mathrm{m}$ versus $200-\mu \mathrm{m}$ EX-PRESS device in humans. In our study, both devices were equally beneficial.

In conclusion, our experience indicates that the EX-PRESS filtration device has a good and long-lasting IOP-lowering effect with a low rate of mild and transient complications in patients with NVG due to PDR. 
Hanna et al.: Clinical Efficacy and Safety of the EX-PRESS Filtration Device in Patients with

Advanced Neovascular Glaucoma and Proliferative Diabetic Retinopathy

\section{Statement of Ethics}

The study was approved by the Hillel Yaffe Medical Center's Institutional Review Board.

\section{Disclosure Statement}

The authors report no conflict of interest with this work.

\section{References}

1 Sivak-Callcott JA, O'Day DM, Gass DM, Tsi JC: Evidence-based recommendations for the diagnosis and treatment of neovascular glaucoma. Ophthalmology 2001;108:1767-1778.

Brown GC, Magargal LE, Schachat A, Shah H: Neovascular glaucoma. Etiologic considerations. Ophthalmology 1984;91:315-320.

Casey R, Li WW: Factors controlling ocular angiogenesis. Am J Ophthalmol 1997;124:521-529.

Tripathi RC, Li J, Tripathi BJ, Chalam KV, Adamis AP: Increased level of vascular endothelial growth factor in aqueous humor of patients with neovascular glaucoma. Ophthalmology 1998;105:232-237. Chen KH, Wu CC, Roy S, Lee SM, Liu JH: Increased interleukin-6 in aqueous humor of neovascular glaucoma. Invest Ophthalmol Vis Sci 1999;40:2627-2632. Allen RC, Bellows AR, Hutchinson BT, et al: Filtration surgery in the treatment of neovascular glaucoma. Ophthalmology 1982;89:1181-1187.

7 Hong $\mathrm{CH}$, Arosemena A, Zurakowski D, et al: Glaucoma drainage devices: a systematic literature review and current controversies. Surv Ophthalmol 2005;50:48-60.

8 Yalvac IS, Eksioglu U, Satana B, et al: Long-term results of Ahmed valve and Molteno implant in neovascular glaucoma. Eye 2007;21:65-70. Ayyala RS, Zurakowski D, Smith JA, et al: A clinical study of the Ahmed glaucoma valve implant in advanced glaucoma. Ophthalmology 1998;105:1968-1976.

Huang MC, Netland PA, Coleman AL, et al: Intermediate term clinical experience with the Ahmed Glaucoma Valve implant. Am J Ophthalmol 1999;127:27-33.

-11 Valimaki J, Tuulonen A, Airaksinen PJ: Outcome of Molteno implantation surgery in refractory glaucoma and the effect of total and partial tube ligation on the success rate. Acta Ophthalmol Scand 1998;76:213-219.

Coleman AL, Hill R, Wilson MR, et al: Initial clinical experience with the Ahmed Glaucoma Valve implant. Am J Ophthalmol 1995;120:23-31.

13 Dahan E, Carmichael TR: Implantation of a miniature glaucoma device under a scleral flap. J Glaucoma 2005;14:98-102.

14 Wamsley S, Moster MR, Rai S, et al: Results of the use of the EX-PRESS miniature glaucoma implant in technically challenging, advanced glaucoma cases: a clinical pilot study. Am J Ophthalmol 2004;138:1049-1051.

15 Parekh AS, Tafreshi A, Dorairaj SK, Weinreb RN: Clinical applicability of the International Classification of Disease and Related Health Problems (ICD-9) glaucoma staging codes to predict disease severity in patients with open-angle glaucoma. J Glaucoma 2014;23:e18-e22.

16 Mahdy RA, Nada WM, Fawzy KM, et al: Efficacy of intravitreal bevacizumab with panretinal photocoagulation followed by Ahmed valve implantation in neovascular glaucoma. J Glaucoma 2013;22:768-772.

17 Ma KT, Yang JY, Kim JH, et al: Surgical results of Ahmed valve implantation with intraoperative bevacizumab injection in patients with neovascular glaucoma. J Glaucoma 2012;21:331-336.

18 Sidoti PA, Dunphy TR, Baerveldt G, et al: Experience with the Baerveldt glaucoma implant in treating neovascular glaucoma. Ophthalmology 1995;102:1107-1118.

19 Mermoud A, Salmon JF, Alexander P, et al: Molteno tube implantation for neovascular glaucoma. Longterm results and factors influencing the outcome. Ophthalmology 1993;100:897-902.

20 Every SG, Molteno ACB, Bevin TH, et al: Long-term results of Molteno implant insertion in cases of neovascular glaucoma. Arch Ophthalmol 2006;124:355-360. 
Hanna et al.: Clinical Efficacy and Safety of the EX-PRESS Filtration Device in Patients with Advanced Neovascular Glaucoma and Proliferative Diabetic Retinopathy

Dahan E: EX-PRESS miniature glaucoma implant. Expert Rev Ophthalmol 2007;2:899-909.

Hendrick AM, Kahook MY: EX-PRESS mini glaucoma shunt: surgical technique and review of clinical experience. Expert Rev Med Devices 2008;5:673-678.

23 Ayyala RS, Zurakowski D, Monshizadeh R, et al: Comparison of double-plate Molteno and Ahmed glaucoma valve in patients with advanced uncontrolled glaucoma. Ophthalmic Surg Lasers 2002;33:94101.

24 Wakabayashi T, Oshima Y, Sakaguchi H, et al: Intravitreal bevacizumab to treat iris neovascularization and neovascular glaucoma secondary to ischemic retinal diseases in 41 consecutive cases. Ophthalmology 2008;115:1571-1580.

25 Ehlers JP, Spirn MJ, Lam A, et al: Combination intravitreal bevacizumab/panretinal photocoagulation versus panretinal photocoagulation alone in the treatment of neovascular glaucoma. Retina 2008;28:696-702.

Drs. Hanna and Tiosano contributed equally to this study.

40.0

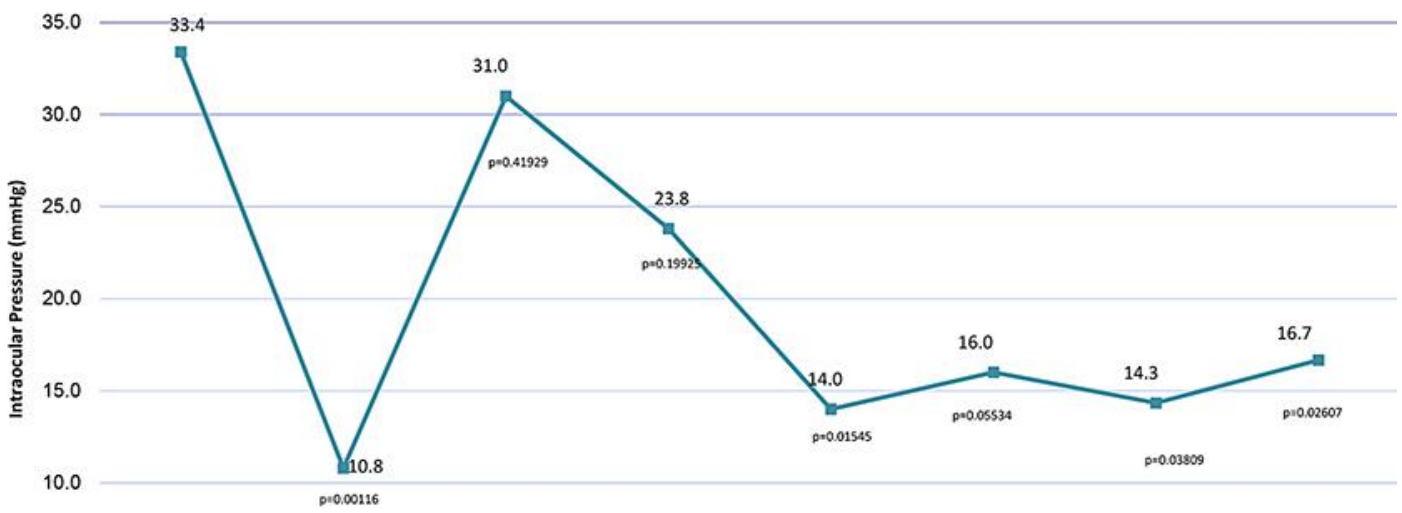

5.0

0.0

PRE OP day 1 months $0-1$ months 2-3 months 4.6

Fig. 1. Surgical outcome at the last follow-up. 


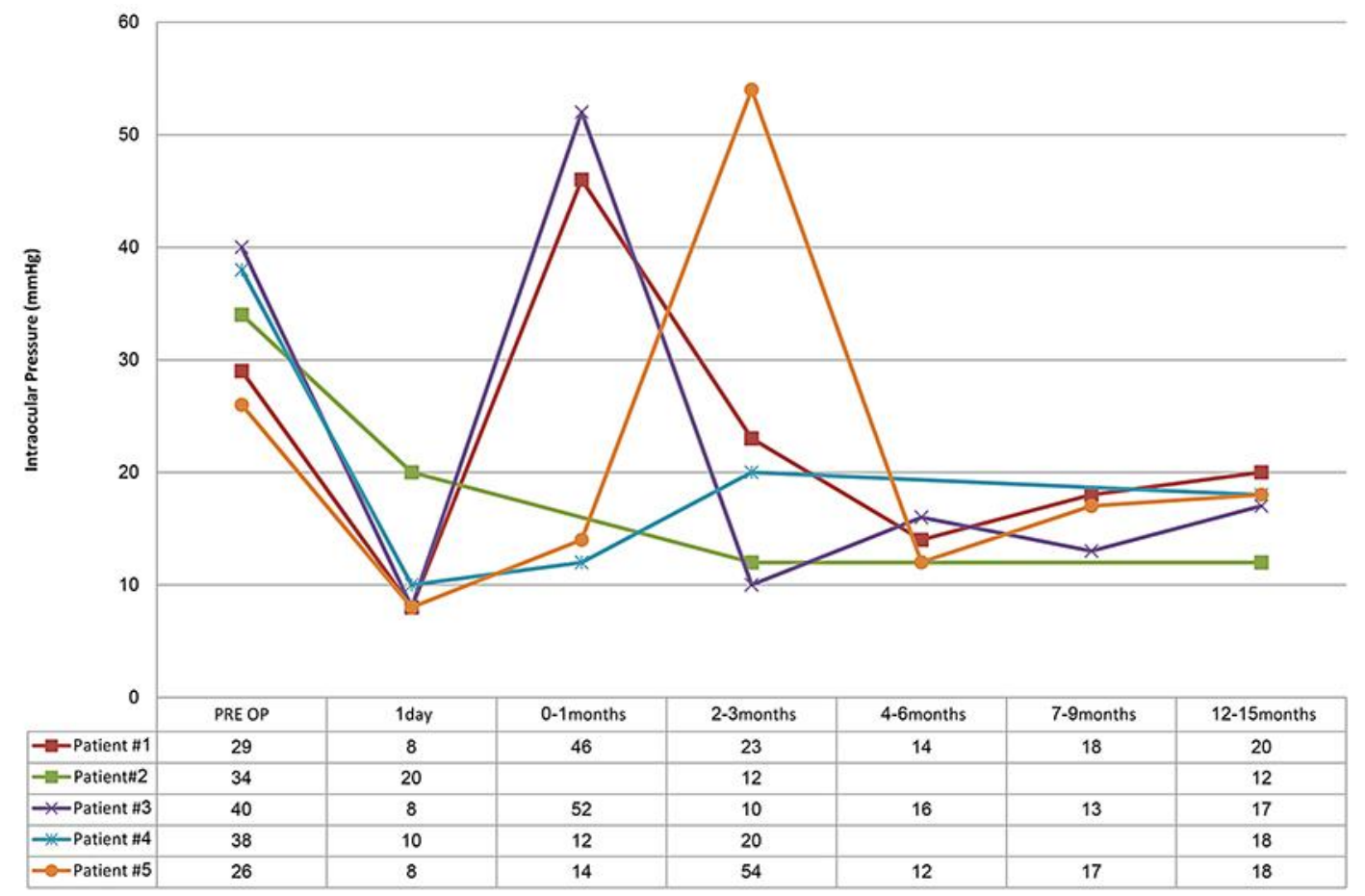

Fig. 2. Intraocular pressure measurements for each patient until final follow-up.

Table 1. Baseline characteristics of the study group

Male:female

Mean age \pm SD, years

Side of operated eye

Right:left

Phakia:pseudophakia

Prior glaucoma surgery

Prior vitrectomy

Prior PRP laser

Prior intravitreal bevacizumab injection (c) 2018 The Author(s). Published by S. Karger AG, Basel www.karger.com/cop

Hanna et al.: Clinical Efficacy and Safety of the EX-PRESS Filtration Device in Patients with Advanced Neovascular Glaucoma and Proliferative Diabetic Retinopathy

PRP, panretinal photocoagulation; SD, standard deviation. 
Hanna et al.: Clinical Efficacy and Safety of the EX-PRESS Filtration Device in Patients with Advanced Neovascular Glaucoma and Proliferative Diabetic Retinopathy

Table 2. Comparison of preoperative and postoperative results with the EX-PRESS glaucoma device in neovascular glaucoma

\begin{tabular}{lccc}
\hline & Preoperative & Postoperative & $p$ value \\
\hline Mean IOP \pm SD, mm Hg & $33.4 \pm 5.9$ & $17.0 \pm 3.0$ & 0.00368 \\
Mean number of glaucoma medications \pm SD & $3.8 \pm 0.4$ & $2.2 \pm 1.5$ & 0.06012 \\
Visual acuity, $n$ patients & & & \\
$\quad 20 / 200$ & 2 & 2 & \\
$\quad<20 / 200-C F$ & 1 & 1 & \\
Hand motion and light perception & 1 & & \\
\hline
\end{tabular}

IOP, intraocular pressure; SD, standard deviation. 